\title{
Improved fuzzy logic method to distinguish between meteorological and non-meteorological echoes using C-band polarimetric radar data
}

\author{
Shuai Zhang ${ }^{1}$, Xingyou Huang ${ }^{1}$, Jinzhong Min ${ }^{1}$, Zhigang Chu ${ }^{1}$, Xiaoran Zhuang ${ }^{1}$, and Hengheng Zhang ${ }^{2}$ \\ ${ }^{1}$ Key Laboratory of Meteorological Disaster of Ministry of Education, Nanjing University of Information \\ Science and Technology, Nanjing, China \\ ${ }^{2}$ Institute of Meteorology and Climate Research, Karlsruhe Institute of Technology, Karlsruhe, Germany
}

Correspondence: Xingyou Huang (huangxy@nuist.edu.cn)

Received: 6 September 2019 - Discussion started: 20 September 2019

Revised: 28 December 2019 - Accepted: 10 January 2020 - Published: 7 February 2020

\begin{abstract}
To obtain better performance of meteorological applications, it is necessary to distinguish radar echoes from meteorological and non-meteorological targets. After a comprehensive analysis of the computational efficiency and radar system characteristics, we propose a fuzzy logic method that is similar to the MetSignal algorithm; the performance of this method is improved significantly in weak-signal regions where polarimetric variables are severely affected by noise. In addition, post-processing is adjusted to prevent anomalous propagation at a far range from being misclassified as meteorological echo. Moreover, an additional fuzzy logic echo classifier is incorporated into post-processing to suppress misclassification in the melting layer. An independent test set is selected to evaluate algorithm performance, and the statistical results show an improvement in the algorithm performance, especially with respect to the classification of meteorological echoes in weak-signal regions.
\end{abstract}

\section{Introduction}

Weather radar with dual-polarization capability has a wider range of application than conventional weather radar (i.e., single-polarization weather radar), in terms of providing information regarding the shape, size, spatial orientation, and physical composition of hydrometeors (Kumjian, 2013a, b, c). Significant improvements have been made in meteorological and hydrological applications (e.g., data assimilation, quantitative precipitation estimation, and hydrome- teor classification) after using polarimetric radar data (Giangrande and Ryzhkov, 2008; Jung et al., 2008a, b; Park et al., 2009). However, the existence of non-meteorological echoes (NMETs; e.g., ground clutter - GC; anomalous propagation - AP; clear-air echoes - CA) in radar data often reduces the application performance. Therefore, it is necessary to separate radar data that contain meteorological echoes (METs; e.g., rain, snow, and hail) from those that contain NMET, before these applications are implemented.

Several effective algorithms for distinguishing between NMET and MET have been proposed in recent years. Lakshmanan et al. (2014) developed an algorithm based on neural networks for radar data quality control. The raw values and local variance of polarimetric variables and Doppler moments, as well as features calculated from a 3-D virtual volume, are selected as neural network inputs. The output of the neural network is the MET probability at each range gate. The range gates are then clustered into contiguous regions, and the probabilities are averaged within each cluster. The average probability is compared with a preset probability threshold to determine whether the cluster is retained (considered as MET) or censored (considered as NMET). A MET-NMET classifier was developed by Tang et al. (2014) to perform reflectivity data quality control using polarimetric radar variables and atmospheric environmental data. The algorithm combines a simple correlation coefficient filter as the primary determinant and applies a set of physically based rules to handle some special MET (e.g., hail, non-uniform beam filling, and melting layer - ML) and NMET (e.g., 
random clutter with a high correlation coefficient). Krause (2016) proposed an algorithm, MetSignal, to distinguish between MET and NMET using polarimetric radar data, which has a simple design and allows users to adjust its performance according to a specific situation. The MetSignal algorithm is based on a fuzzy logic technique with a few postprocessing rules; it has been selected to be implemented on the WSR-88D network in the United States. In addition, the performance of different methods in the context of distinguishing between MET and NMET is compared in RicoRamirez and Cluckie (2008) and Islam et al. (2012). Further, the importance of different features is also evaluated by Lakshmanan et al. (2015).

Compared with the other two methods (Tang et al., 2014; Krause, 2016), the most obvious disadvantage of the neural network method proposed by Lakshmanan et al. (2014) is the heavy computation intensity; this renders it unsuitable for operational applications, especially for radar systems with a high spatial and/or temporal resolution. Although the method proposed by Tang et al. (2014) has a higher computational efficiency, it may result in undesirable performance if applied in polarimetric radar systems with imperfect hardware technology or without noise correction (Gourley et al., 2006; Schuur et al., 2003), which is primarily attributed to excessive dependence on the correlation coefficient. Fuzzy logic is a multiple-input classifier method that can minimize the impact from a single erroneous input. In addition, the MetSignal algorithm has the highest computational efficiency among the three methods (Krause, 2016; Tang et al., 2014). Therefore, the framework of the MetSignal algorithm is adopted in this paper.

Like most methods in the context of distinguishing between MET and NMET based on polarimetric radar data, the MetSignal algorithm has high expectations for polarimetric features and sets high weights for them. However, the fluctuation of polarimetric variables is very violent in the weak-signal regions and regions affected by ML, which is not conducive to the purpose of distinguishing MET and NMET (Krause, 2016; Rico-Ramirez and Cluckie, 2008). The suppression of misclassification in ML regions is included in the post-processing of the MetSignal algorithm; however, the necessary consideration is lacking in weak-signal regions, where polarimetric variables are severely affected by noise. The main purpose of the improved method proposed in this paper is to improve the performance of the MetSignal algorithm in weak-signal regions, hence referred as "MetSignal_noise". Additional adjustments and improvements over the MetSignal algorithm are also included in the MetSignal_noise algorithm.

The rest of this paper is organized as follows. Section 2 briefly describes the radar system used in this study and the available measurements. Subsequently, a detailed explanation of the proposed algorithm is provided in Sect. 3, and Sect. 4 presents the algorithm performance evaluation results. Finally, conclusions are provided in Sect. 5.
Table 1. Main parameters of NUIST-CDP.

\begin{tabular}{ll}
\hline Parameters & NUIST-CDP \\
\hline Transmitter & Klystron $(5600 \mathrm{MHz})$ \\
Pulse width & $0.5 \mu \mathrm{m}$ \\
Pulse repetition frequency & $1000 \mathrm{~Hz}$ \\
Peak power & $250 \mathrm{~kW}$ \\
\hline Receiver & Simultaneous horizontal/vertical \\
Noise figure & $3 \mathrm{~dB}$ \\
Dynamic range & $90 \mathrm{~dB}$ \\
Sensitivity & $-109 \mathrm{dBm}$ \\
\hline Antenna feeder & Paraboloid \\
Antenna gain & $48.5 \mathrm{~dB}$ \\
Reflector diameter & $8.5 \mathrm{~m}$ \\
Beam width & $0.54^{\circ}$ \\
\hline
\end{tabular}

\section{Instrument and data}

The radar data used in this study were collected by a Cband dual-polarization Doppler weather radar owned by the Nanjing University of Information Science and Technology (NUIST-CDP). The main parameters of NUIST-CDP are listed in Table 1. NUIST-CDP is designed and manufactured by Beijing Metstar Radar Company in China and is deployed at the university campus $\left(32.21^{\circ} \mathrm{N}, 118.72^{\circ} \mathrm{E}\right)$. The routine scanning mode of NUIST-CDP is set to volume scanning with 14 elevation angles $(0.5,1.5,2.4,3.4,4.3,5.3,6.2$, $7.5,8.7,10,12,14,16.7$, and $19.5^{\circ}$ ) at a 7 min scan update rate. The available measurements include the reflectivity factor at the horizontal polarization $(Z)$, Doppler velocity $(V)$, Doppler spectrum width $(W)$, differential reflectivity (ZDR), differential propagation phase shift (PhiDP), co-polar correlation coefficient (CC), signal-to-noise ratio (SNR), and signal quality index (SQI); all of these are at a radial range resolution of $75 \mathrm{~m}$.

The NUIST-CDP data are seriously affected by GC and $\mathrm{AP}$, which is attributed to the absence of clutter filtering in the signal processing. The strong CA is one of the main sources of error for some meteorological and hydrological applications (Stumpf et al., 1998; Zhang et al., 2011), which often appears in the NUIST-CDP data during the warm season. In addition, NUIST-CDP has a higher pulse repetition frequency than the operational radar (Crum and Alberty, 1993), which implies the existence of a shorter maximum detection range and more frequent second-trip echoes. Considering that the second-trip echo is formed by meteorological targets, the algorithm temporarily classifies it as MET. The identification and removal of second-trip echoes will be considered in future research. 


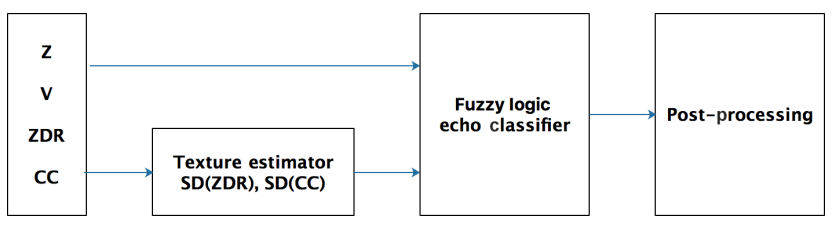

Figure 1. Block diagram of the MetSignal algorithm.

\section{Method}

\subsection{MetSignal}

Since a similar algorithm framework is used in both the MetSignal and the MetSignal_noise algorithms, a brief description of the MetSignal algorithm is presented first. Figure 1 summarizes the major steps of the MetSignal algorithm as a block diagram.

Except for the raw radar variables (i.e., $Z, V$, and CC), two texture parameters (i.e., $\mathrm{SD}(\mathrm{ZDR})$ and $\mathrm{SD}(\mathrm{CC})$ ) are also input as features into the fuzzy logic echo classifier. SD(ZDR) and $\mathrm{SD}(\mathrm{CC})$ are estimated by calculating standard deviations of ZDR and CC along a radial for 21 range gates $(1.5 \mathrm{~km}$ in NUIST-CDP) centered on the target gate, which can characterize the magnitude of small-scale fluctuations in ZDR and CC. It is worth noting that the $\mathrm{SD}$ (PhiDP) input in the raw version of the MetSignal algorithm was removed to avoid texture estimation errors because of phase folding. Although there are some conventional methods to solve phase folding (Wang and Chandrasekar, 2009), they fail when applied to radar data mixed with first- and second-trip echoes.

The fuzzy logic technique is adopted in the echo classifier, which is a classification methodology widely used in the weather radar community (Gourley et al., 2007; Lin et al., 2012; Liu and Chandrasekar, 2000). The additive method is applied in the MetSignal algorithm to obtain the aggregation value for MET $\left(A_{\mathrm{MET}}\right)$ to maximize the probability of detection (the multiplicative method is another way which aims to minimize false classification; Zrnić et al., 2001). The $A_{\text {MET }}$ is computed as

$A_{\mathrm{MET}}=\frac{\sum W_{x} \mathrm{MF}_{x}}{\sum W_{x}}$

where $x$ is one of the five features mentioned above and $W_{x}$ and $\mathrm{MF}_{x}$ are the weights (the weight setting for each feature is shown in Table 2) and membership function value of $x$, respectively.

For the classification method using fuzzy logic, the membership functions selection often determines the final classification performance to a certain extent. Considering that the characteristics of radar variables depend on the specific radar systems as well as the climatological and geographical location of the radar, the membership functions are objectively determined by the statistical analysis of the NUISTCDP measured data. Table 3 summarizes the data used for
Table 2. The weight setting for each feature in the MetSignal algorithm.

\begin{tabular}{lc}
\hline Feature & Weight \\
\hline$Z$ & 1 \\
$V$ & 1 \\
CC & 1 \\
SD(ZDR) & 2 \\
SD(CC) & 1 \\
\hline
\end{tabular}

Table 3. List of events used for training membership functions (UTC).

\begin{tabular}{ll}
\hline Date & Description \\
\hline 00:00-01:00 1 May 2017 & AP \\
01:00-02:00 2 May 2017 & Stratiform precipitation \\
04:00-05:00 3 May 2017 & CA \\
14:00-15:00 3 May 2017 & Convective precipitation \\
18:00-19:00 4 May 2017 & GC \\
20:00-21:00 5 May 2017 & Stratiform precipitation \\
00:00-01:00 6 May 2017 & GC \\
08:00-09:00 6 May 2017 & Stratiform precipitation \\
06:00-07:00 7 May 2017 & CA \\
18:00-19:00 7 May 2017 & Convective precipitation \\
22:00-23:00 8 May 2017 & GC \\
01:00-02:00 11 May 2017 & AP \\
09:00-10:00 11 May 2017 & Convective precipitation \\
17:00-18:00 11 May 2017 & Stratiform precipitation \\
13:00-14:00 12 May 2017 & CA \\
22:00-23:00 13 May 2017 & AP \\
06:00-07:00 14 May 2017 & Convective precipitation \\
17:00-18:00 14 May 2017 & Stratiform precipitation \\
01:00-02:00 15 May 2017 & CA \\
22:00-23:00 16 May 2017 & AP \\
14:00-15:00 18 May 2017 & CA \\
09:00-10:00 19 May 2017 & Stratiform precipitation \\
08:00-09:00 20 May 2017 & Convective precipitation \\
\hline
\end{tabular}

training, which are manually extracted by experienced meteorologists through a simple graphical user interface and consist of several typical events; these include GC, AP, CA, stratiform precipitation, and convective precipitation. It is worth mentioning that because there is not enough observation data as evidence, the training set does not include extremely complex situations (e.g., the boundary or transition zone between MET and NMET) to prevent the introduction of subjective bias.

The normalized frequency distributions of the features are shown in Fig. 2, which are derived using the training set. The method proposed by Cho et al. (2006) is used to determine the membership functions; it has a higher efficiency than the iterative method used in Krause (2016). The $\mathrm{MF}_{x}$ is com- 

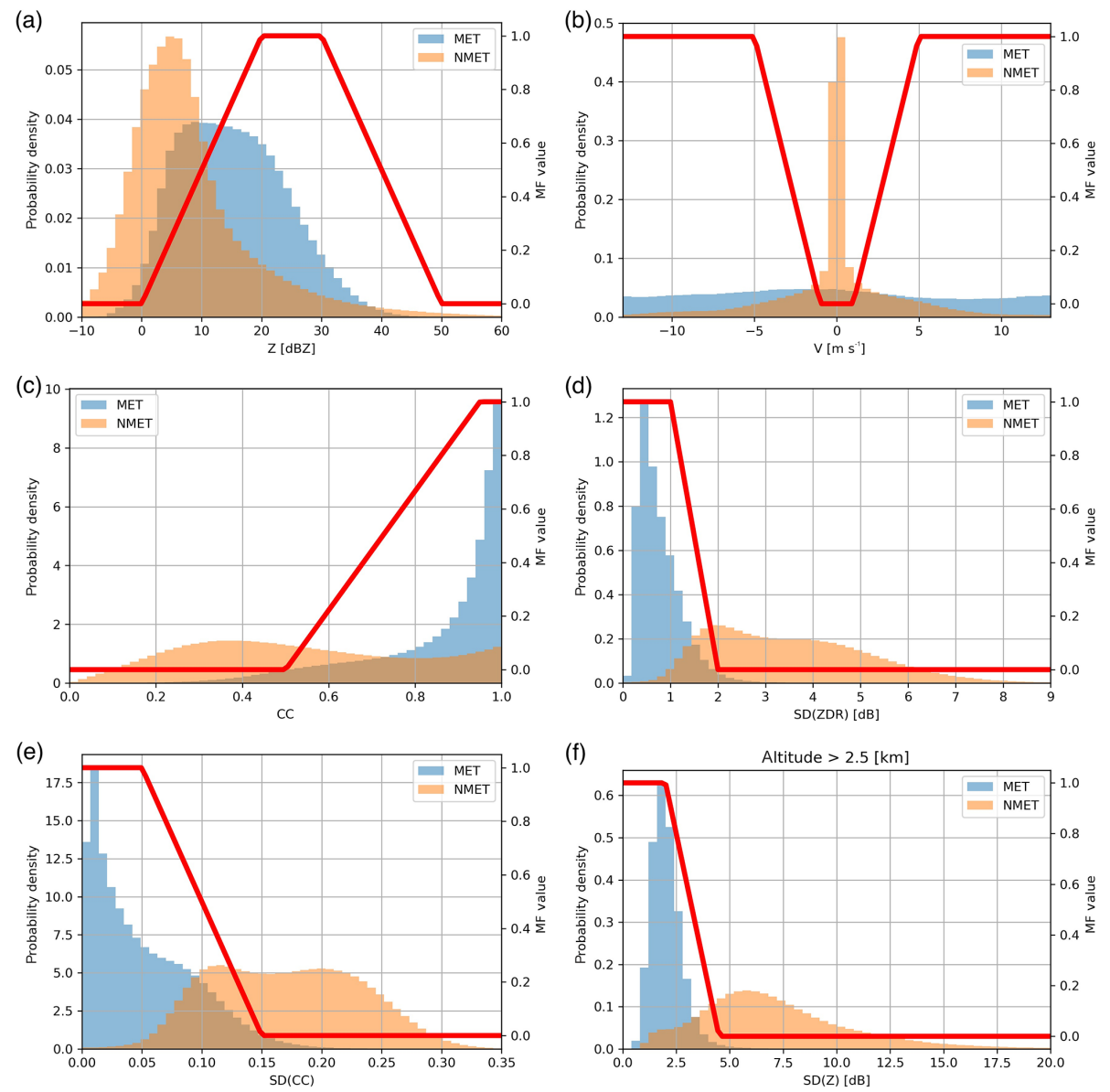

Figure 2. The normalized frequency distributions and membership functions of features: (a) $Z$, (b) $V$, (c) $\mathrm{CC}$, (d) $\mathrm{SD}(\mathrm{ZDR})$, (e) $\mathrm{SD}(\mathrm{CC})$, and (f) $\mathrm{SD}(Z)$ (in the regions over $2.5 \mathrm{~km}$ in height).

puted as

$\mathrm{MF}_{x}=\frac{\mathrm{FMET}_{x}}{\operatorname{FMET}_{x}+\mathrm{FNMET}_{x}}$,

where FMET and FNMET are the normalized frequencies of MET and NMET. The trapezoidal functions are adopted to indicate the membership functions by fitting the results of Eq. (2) using the least-squares method (the red lines in Fig. 2).

After getting the $A_{\mathrm{MET}}$ by the calculation of Eq. (1), we compared it to a preset threshold. The target gate will be classified as MET if $A_{\mathrm{MET}}$ exceeds the threshold; otherwise, it will be classified as NMET. Similar to membership functions, this threshold is also local, needing statistical analysis to get the optimal result. The normalized frequency distributions of $A_{\mathrm{MET}}$, which are derived using the training set, are shown in Fig. 3a. It can be seen that there is a certain degree of overlap between the distribution of MET and NMET, and an obvious intersection is at about 0.5 (the red lines in Fig. 3a). Therefore, 0.5 can be considered as an optimal $A_{\text {MET }}$ threshold of the MetSignal algorithm on NUIST-CDP.
After obtaining the preliminary results of the fuzzy logic echo classifier, a set of post-processing rules are adopted in the MetSignal algorithm to adjust the classification results appropriately to make them more reasonable. These rules include a ZDR filter for eliminating residual CA (the range gates with an absolute value of ZDR exceeding $4.5 \mathrm{~dB}$ are considered as NMET), a CC filter (the range gates with CC less than 0.65 are considered as NMET), and forced classification as MET will also be performed in range gates where $Z$ at a height of $3 \mathrm{~km}$ in the previous volume scan at the same location exceeds $11 \mathrm{dBZ}$, which will help to prevent misclassification in ML regions. A typical case of CA (02:23 UTC on 7 May 2017) shown in Fig. 4 can well demonstrate the need for post-processing (take the ZDR filter as an example). In the field of $A_{\mathrm{MET}}$ (Fig. $4 \mathrm{~b}$ ), many regions exceed the threshold (0.5), which will cause CA to be misclassified as MET. The primary reason for this problem is that $\mathrm{CA}$ in these regions has a relatively uniform ZDR and small SD(ZDR) (Fig. 4c and d), which causes an incorrect increase of $\mathrm{MF}_{\mathrm{SD}(\mathrm{ZDR})}$ and $A_{\mathrm{MET}}$. Compared with the classification result directly based on the output of the fuzzy logic echo 

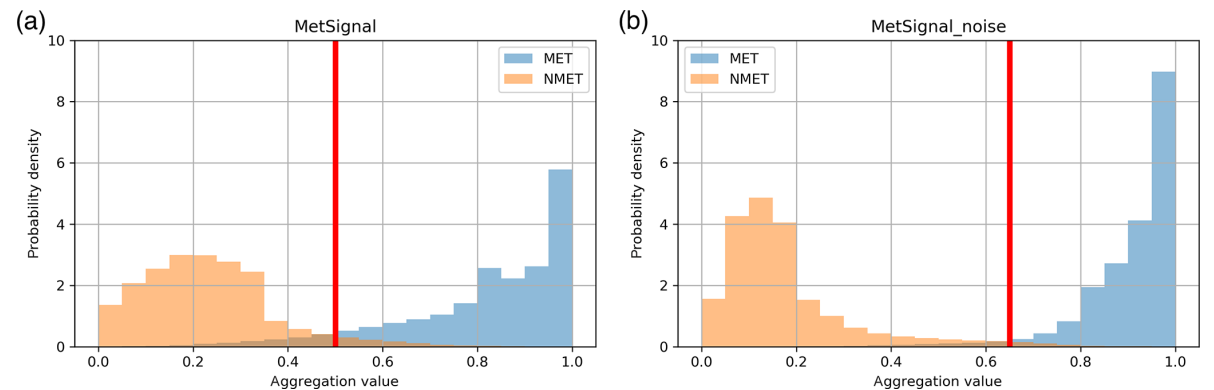

Figure 3. The normalized frequency distributions and thresholds of $A_{\mathrm{MET}}$

(a) MetSignal and (b) MetSignal_noise.

classifier (Fig. 4e), the misclassification is effectively suppressed after post-processing (Fig. 4f).

\subsection{Improvements and adjustments in MetSignal_noise}

\subsubsection{The limitation of the use scope of $V$}

As shown in Fig. 2b, although the $V$ of NMET is mainly concentrated near $0 \mathrm{~m} \mathrm{~s}^{-1}$, while the $V$ of MET is uniformly distributed in the whole range, there is still a large overlap between them in the regions where the absolute value of $V$ is large. The broadening of the NMET frequency distribution is mainly attributed to the existence of CA, which is similar to that of MET in terms of $V$ (Wilson et al., 1994). Considering that the $V$ does not play a role in distinguishing MET from CA, some constraint conditions should be set to limit the use scope of $V$ in the fuzzy logic echo classifier. Since the CA usually has a smaller $Z$ and larger $W$ than GC and AP (Fang et al., 2004; Wilson et al., 1994), a 2-D histogram method is adopted to analyze the NMET's $V$ vs. $Z$ and $V$ vs. $W$ relationships in the training set to find the thresholds of $Z$ and $W$ for separating CA from other NMET instances, as far as possible. As shown in Fig. 5a, the 2-D histogram of $V$ vs. $Z$ of NMET presents an orthogonal shape, which is composed of GC and AP with $V$ approximately equal to $0 \mathrm{~m} \mathrm{~s}^{-1}$ and CA with $Z$ below $30 \mathrm{dBZ}$ and $V$ uniformly distributed in the whole range. As shown in Fig. 5b, the 2-D histogram of $V$ vs. $W$ of NMET is uniform overall, except for the region where $V$ is close to $0 \mathrm{~m} \mathrm{~s}^{-1}$ and $W$ is less than $2 \mathrm{~m} \mathrm{~s}^{-1}$. This region is very concentrated and should be composed of GC and AP due to its static and stable characteristics. Therefore, $V$ is used in fuzzy logic echo classifier as a feature only when $Z$ is greater than $30 \mathrm{dBZ}$ or $W$ is less than $2 \mathrm{~m} \mathrm{~s}^{-1}$. The normalized frequency distribution and membership function of $V$ after setting thresholds of $Z$ and $W$ is shown in Fig. 5c. Compared with Fig. $2 b$, the frequency distribution of NMET in Fig. $5 \mathrm{c}$ is more concentrated at $0 \mathrm{~m} \mathrm{~s}^{-1}$, and the broadening has also been significantly reduced; meanwhile, that of MET remains uniformly distributed in the entire range without any notable changes.

\subsubsection{The decrease of $\mathrm{CC}$ in the region of $\mathrm{GC}$ and $\mathrm{AP}$}

As illustrated in Fig. 2c, there is a significant overlap between NMET and MET in the region where CC is above 0.8 , which increases the difficulty in distinguishing between NMET and MET and is also contrary to the common knowledge that NMET has a low CC (Kumjian, 2013a). After analyzing a large amount of data, it is found that NMET of a high CC mainly comes from GC and AP, which may be due to the characteristics of NUIST-CDP (e.g., spatial resolution and dwell time). Therefore, the method proposed by Zrnić et al. (2006) - CC is averaged along the radial using a 21-range gate window (1.5 km in the NUIST-CDP) - is adopted to reduce CC of NMET with the abnormal high value. As shown in Fig. 6, it is a typical case of AP (23:53 UTC on 24 May 2017) sampled by NUIST-CDP. Compared with the raw CC in Fig. 6b, where some regions of GC and AP have a high $\mathrm{CC}, \mathrm{CC}$ after average processing in Fig. $6 \mathrm{c}$ decreased significantly in these regions and almost all of them were below 0.9 , which was expected to improve the classification performance to some extent.

The distance averaging of CC may produce some undesired side effects in the boundary region between MET and NMET; that is, CC of MET is decreased, while CC of NMET is increased. However, their influence coverage is very limited because the window size is only $1.5 \mathrm{~km}$. In addition, the impact on the averaging results will be further reduced when one of the echo types (MET or NMET) in the window accounts for a large proportion.

\subsubsection{Improvements in weak-signal regions}

As shown in Fig. 7, NUIST-CDP observed a typical case of mixed precipitation accompanied by CA within $50 \mathrm{~km}$ (13:24 UTC on 30 May 2017). A comprehensive analysis of

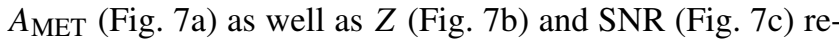
veals that MET with a lower SNR near the echo edge has a lower $A_{\mathrm{MET}}$ than MET in the core regions with a larger SNR, which even close to $A_{\mathrm{MET}}$ of CA. This is because the estimation accuracy of polarimetric variables usually depends on SNR (Bringi and Chandrasekar, 2001). As shown in Fig. 7d and $\mathrm{e}$, a significant fluctuation of ZDR and decrease of CC 
(a) Time: 07-05-2017 02:23:00 UTC

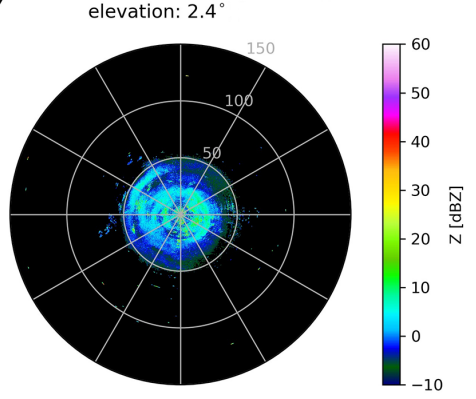

(c) Time: 07-05-2017 02:23:00 UTC

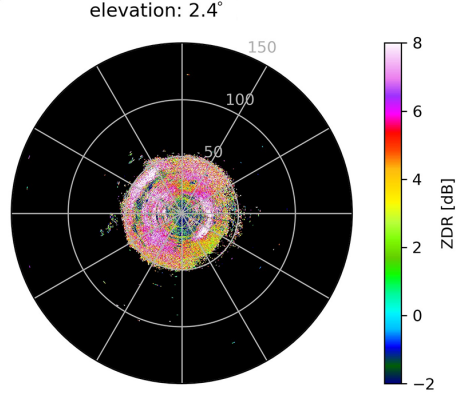

(e) Time: 07-05-2017 02:23:00 UTC

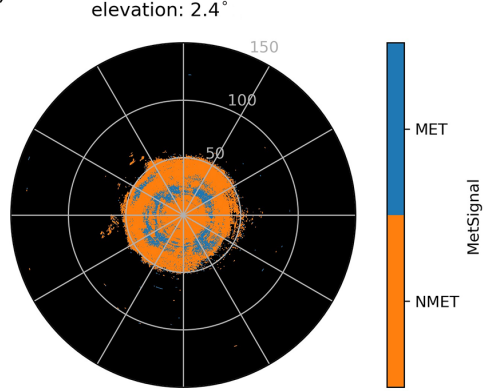

(b) Time: 07-05-2017 02:23:00 UTC

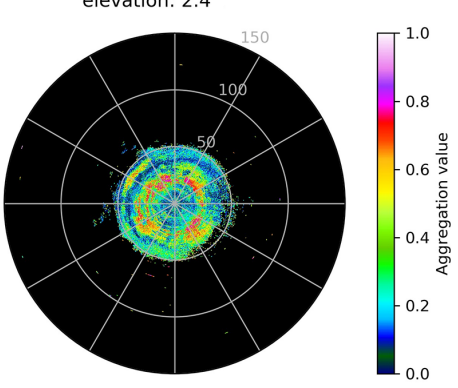

(d) Time: 07-05-2017 02:23:00 UTC

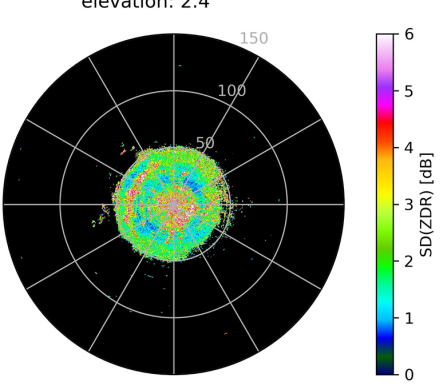

(f) Time: 07-05-2017 02:23:00 UTC

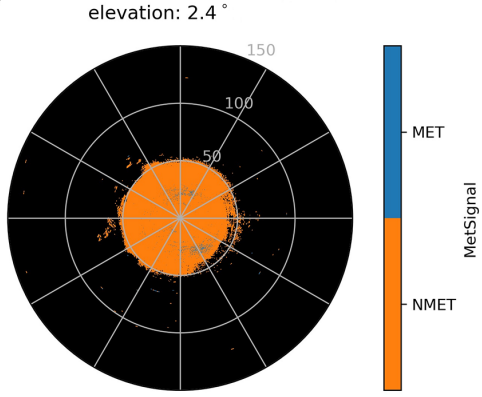

Figure 4. (a) Z, (b) $A_{\mathrm{MET}}$ (MetSignal), (c) ZDR, (d) SD(ZDR), (e) MetSignal before post-processing, and (f) MetSignal after postprocessing. All from NUIST-CDP at 02:23 UTC on 7 May 2017 from an elevation of $2.4^{\circ}$.

(a)

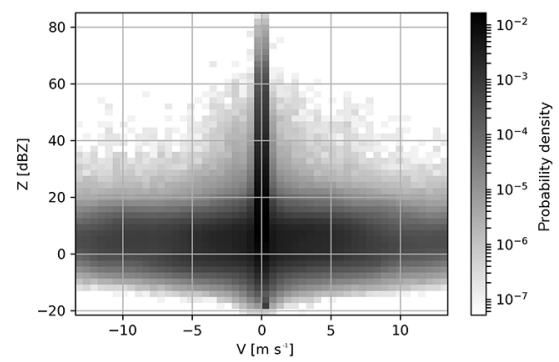

(b)

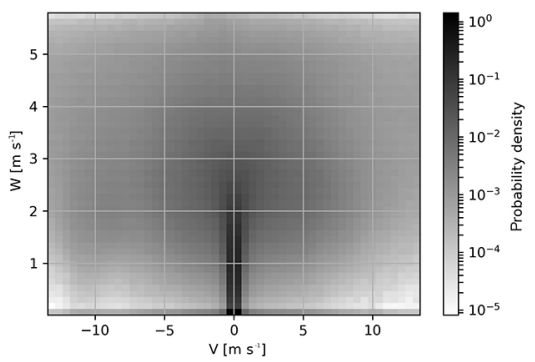

(c)

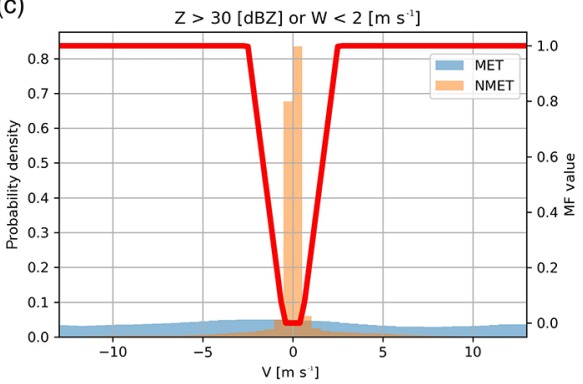

Figure 5. (a) The 2-D histogram of $V$ vs. $Z$ of NMET. (b) The 2-D histogram of $V$ vs. $W$ of NMET. (c) The normalized frequency distribution and membership function of $V$ after setting thresholds of $Z$ and $W$.

can be observed near the echo edge. Meanwhile, their texture (i.e., $\mathrm{SD}(\mathrm{ZDR})$ in Fig. 7f and $\mathrm{SD}(\mathrm{CC})$ in Fig. 7g) has also significantly increased in these regions.

To understand the dependence between polarimetric features and SNR in more detail, a boxplot method is adopted to analyze the MET in the training set. As shown in Fig. 8a, the boxplot of SNR vs. ZDR takes the shape of a dumbbell. The broadening distribution of ZDR with the increase of SNR is attributed to the large raindrops, strong attenuation, and the resonance effect produced by hailstones, which 
(a) Time: 24-05-2017 23:53:48 UTC Elevation: $0.5^{\circ}$

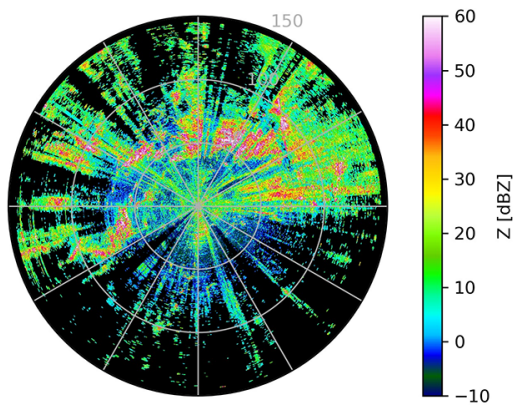

(b) Time: 24-05-2017 23:53:48 UTC Elevation: $0.5^{\circ}$

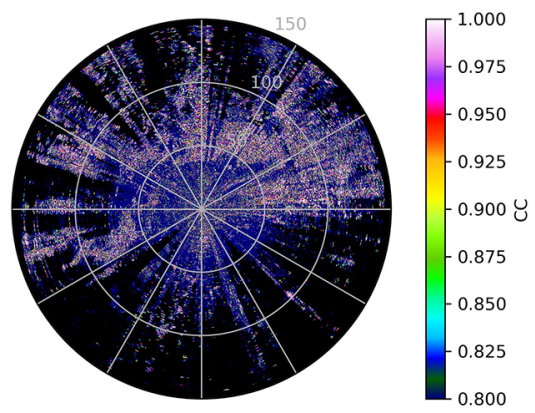

(c) Time: 24-05-2017 23:53:48 UTC

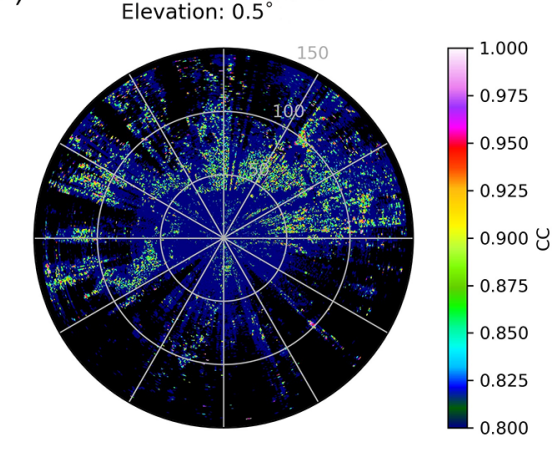

Figure 6. (a) $Z$, (b) CC, and (c) CC after averaging along the radial using a 1.5 km window. All from NUIST-CDP at 23:53 UTC on 24 May 2017 from an elevation of $0.5^{\circ}$.

is easy to be understood and corresponds to common knowledge (Kumjian, 2013c). MET with a smaller SNR usually consists of drizzle, dry snow, and even cloud particles, which should have ZDR close to $0 \mathrm{~dB}$; hence, is often used in ZDR calibration as natural targets (Ryzhkov et al., 2005). Therefore, the ZDR broadening with the decrease of SNR should not be attributed to the microphysical properties of MET, but it should be attributed to the artifacts owing to the influence of noise. Similarly, as shown in Fig. 8b, the magnitude (dispersion) of CC decreases (increases) with a decrease of SNR when SNR is less than $15 \mathrm{~dB}$. These anomalies in ZDR and $\mathrm{CC}$ should be attributed to the weak signal affected by noise, which leads to the polarimetric variables being unable to represent the real microphysical information in MET; further, it also leads to the increase of SD(ZDR) (Fig. 8c) and SD(CC) (Fig. 8d).

Considering the dependence between polarimetric features and SNR, the polarimetric features are stratified by three SNR intervals (less than 5, 5-15, and larger than $15 \mathrm{~dB}$ ), and different processing methods are used for each of these intervals. First, the data of SNR below $5 \mathrm{~dB}$ are directly regarded as noise and not classified. This is because the MET in this interval is extremely affected by noise and is also too weak to play an important role in meteorological and hydrological applications. In addition, as polarimetric variables with a low SNR may increase the texture of adjacent gates, the method proposed by Rico-Ramirez and Cluckie (2008) - masking the polarimetric variables of SNR below $5 \mathrm{~dB}$ in texture calculation - is adopted to reduce the risk of misclassification. Then, the normalized frequency distributions of polarimetric features in Fig. 2 are separated based on different SNR intervals (i.e., 5-15 and larger than $15 \mathrm{~dB}$ ), and the results are shown in Fig. 9. As shown in Fig. 9a and b, the long trailing of CC of MET caused by a low SNR in Fig. 2c is well distinguished from the "normal" MET that has a CC value of approximately 1 and not less than 0.8 . In addition, the odd bimodal distributions of $\mathrm{SD}(\mathrm{ZDR})$ (Fig. 2d) and $\mathrm{SD}(\mathrm{CC})$ (Fig. 2e) are also well decomposed after stratification by SNR (Fig. 9c, d, $\mathrm{e}$, and f), which renders the membership functions more per- tinent, and a better characterization of the polarimetric features is obtained at different SNR intervals.

$A_{\text {MET }}$ obtained by the MetSignal_noise algorithm is shown as Fig. 10a. To better compare the performance of the MetSignal and MetSignal_noise algorithms, $A_{\mathrm{MET}}$ obtained by the MetSignal algorithm with an SNR value less than $5 \mathrm{~dB}$ is masked (Fig. 10b). The difference between them is mainly reflected in the regions of the echo edge and near the radar, which is predominantly contributed by two factors. First, the fluctuation of polarimetric variables is reduced by masking the polarimetric variables of a low SNR (Fig. 10c and d), and the texture of polarimetric variables affected by noise is significantly alleviated (Fig. 10e and f). Second, the polarimetric features can characterize MET and NMET more detailed by adjusting the membership functions based on different SNR intervals (Fig. 9).

Like the method used to determine the $A_{\mathrm{MET}}$ threshold of the MetSignal algorithm, the $A_{\mathrm{MET}}$ threshold of the MetSignal_noise algorithm is set to 0.65 based on the normalized frequency distributions shown in Fig. 3b. Compared with Fig. 3a, the distributions of MET and NMET in Fig. 3b are more focused on both ends, and their overlap is significantly reduced, which can also show that the MetSignal_noise algorithm has a better classification performance than the MetSignal algorithm.

\subsubsection{Post-processing adjustments for ML regions}

The last step in the post-processing of the MetSignal algorithm is to check the constant-altitude plan position indicator (CAPPI) of $Z$ at $3 \mathrm{~km}$ in the previous volume scan. The range gates will be force classified as MET if CAPPI at the same location exceeds $11 \mathrm{dBZ}$; this aims to prevent misclassification in ML regions. However, due to the strong super-refraction caused by specific weather conditions (Doviak and Zrnić, 2006), NUIST-CDP sometimes detects AP with a $Z$ value of more than $11 \mathrm{dBZ}$ at a far range (corresponding to an altitude higher than $3 \mathrm{~km}$ ), which will misclassify AP as MET after post-processing. Figure 11 shows the same AP case as 
(a)

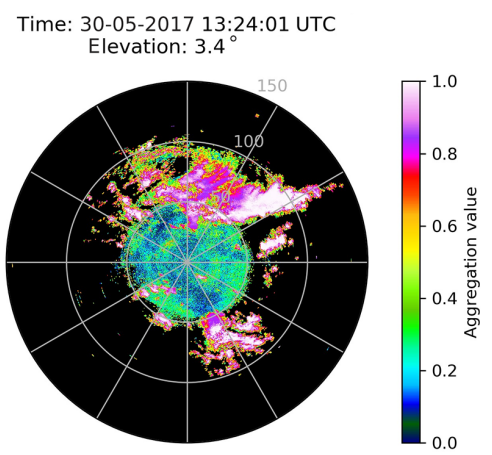

(c) Time: 30-05-2017 13:24:01 UTC

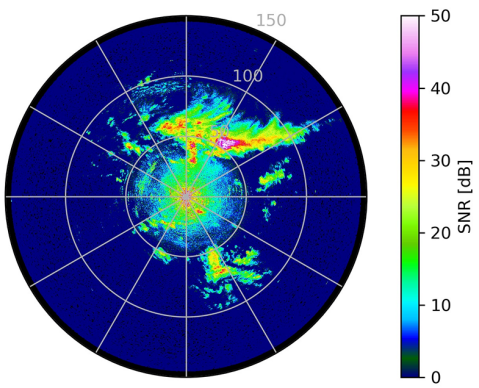

(e) Time: 30-05-2017 13:24:01 UTC

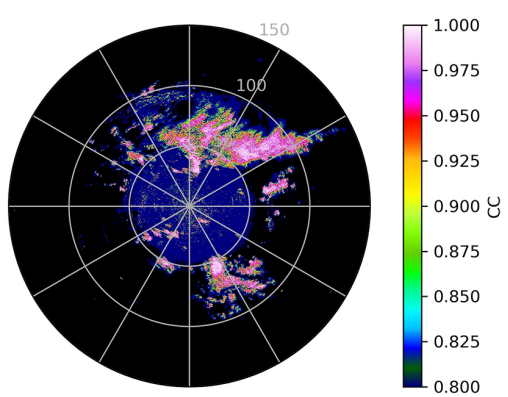

(g) Time: 30-05-2017 13:24:01 UTC

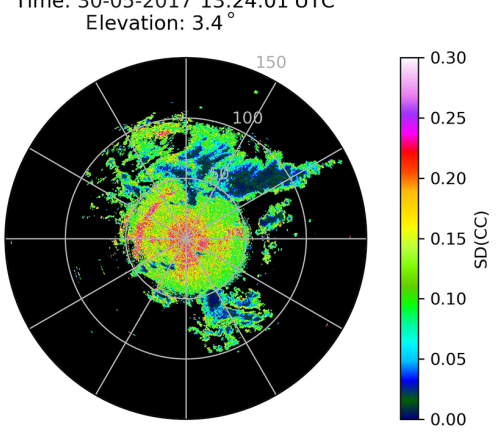

(b) Time: 30-05-2017 13:24:01 UTC

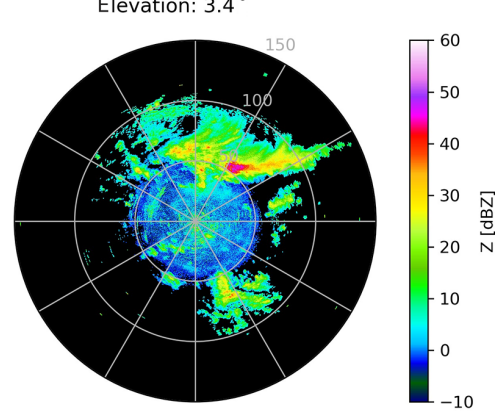

(d) Time: 30-05-2017 13:24:01 UTC

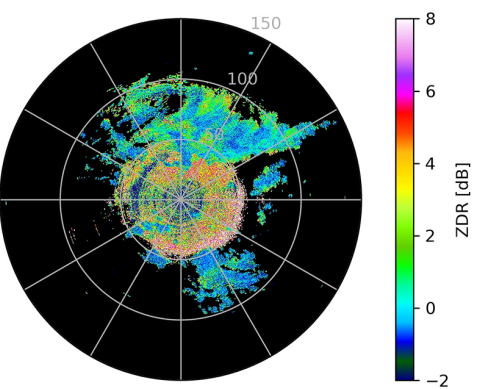

(f) Time: 30-05-2017 13:24:01 UTC

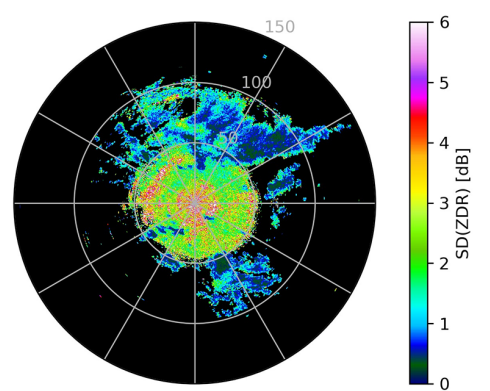

Figure 7. (a) $A_{\mathrm{MET}}$ (MetSignal), (b) Z, (c) SNR, (d) ZDR, (e) CC, (f) SD(ZDR), and (g) SD(CC). All from NUIST-CDP at 13:24 UTC 30 May 2017 from an elevation of $3.4^{\circ}$.

in Fig. 6. Although $A_{\mathrm{MET}}$ obtained by the MetSignal algorithm (Fig. 11a) has a low value (i.e., the classification result is more likely to be NMET), there are still many range gates misclassified as MET in the final result (Fig. 11b) due to improper post-processing. In consideration of the potential risk of misclassifying AP into MET in this post-processing step, this post-processing rule has been removed in the MetSignal_noise algorithm.

However, the lack of special precaution in the ML regions causes a frequent misclassification occurrence because MET 

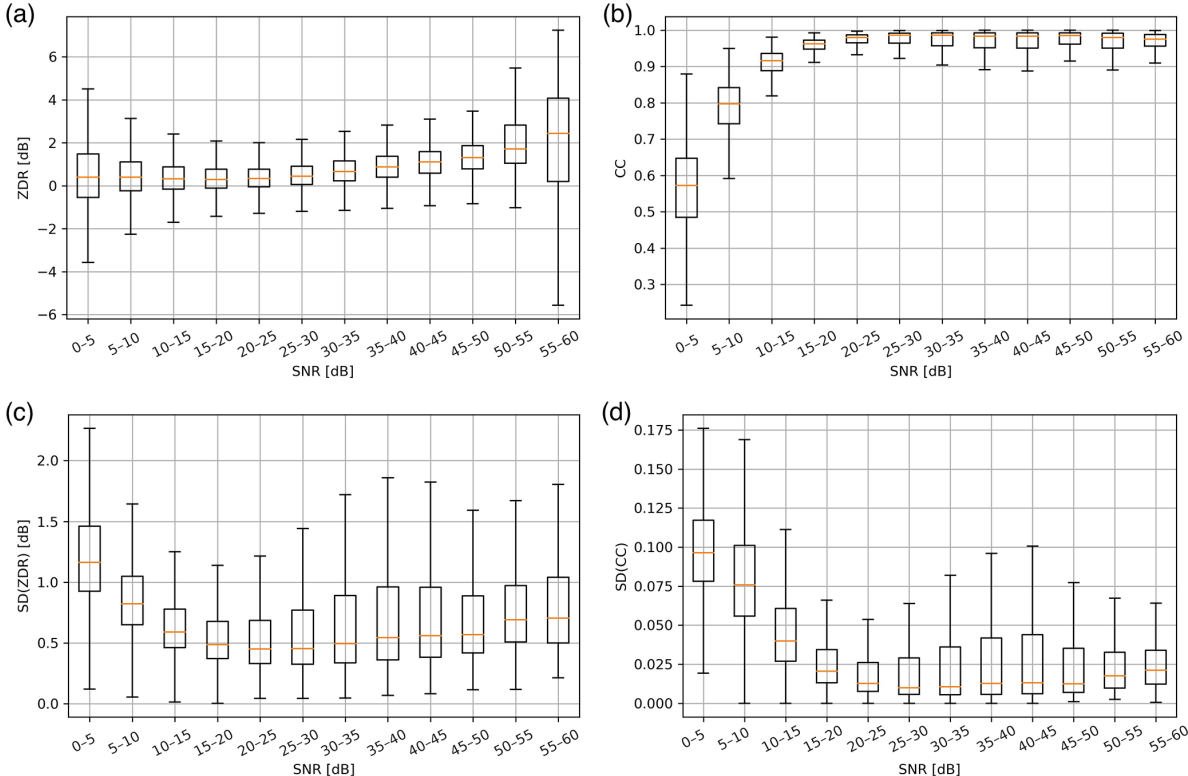

Figure 8. The boxplot of SNR vs. polarimetric features of MET: (a) ZDR, (b) CC, (c) SD(ZDR), and (d) SD(CC).
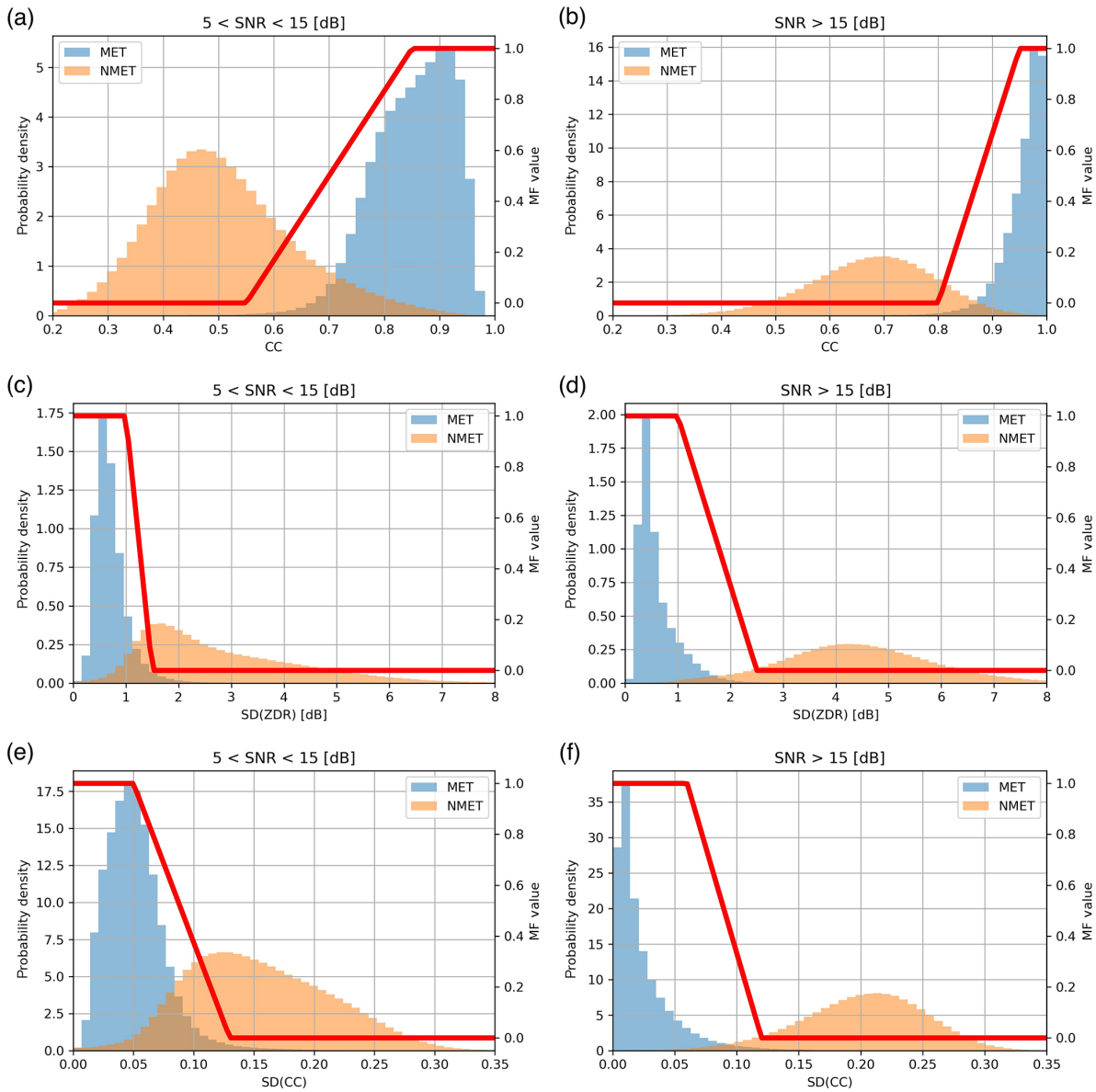

Figure 9. The normalized frequency distributions and membership functions of polarimetric features stratified by SNR: (a) CC $(5<$ SNR $<$ $15 \mathrm{~dB})$, (b) $\mathrm{CC}$ (SNR $>15 \mathrm{~dB})$, (c) $\mathrm{SD}(\mathrm{ZDR})(5<\mathrm{SNR}<15 \mathrm{~dB})$, (d) $\mathrm{SD}(\mathrm{ZDR})$ (SNR $>15 \mathrm{~dB})$, (e) $\mathrm{SD}(\mathrm{CC})(5<\mathrm{SNR}<15 \mathrm{~dB})$, and (f) $\mathrm{SD}(\mathrm{CC})(\mathrm{SNR}>15 \mathrm{~dB})$. 
(a) Time: 30-05-2017 13:24:01 UTC

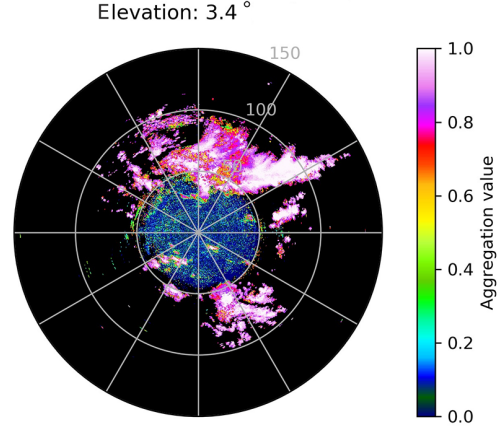

(c) Time: 30-05-2017 13:24:01 UTC

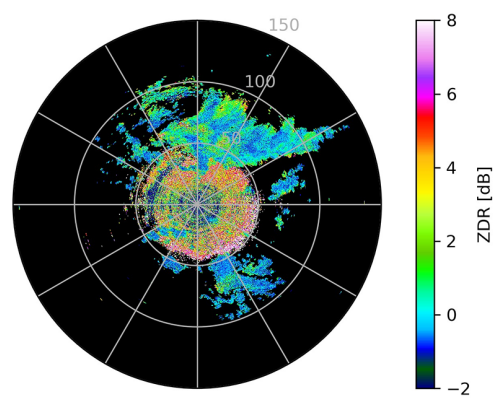

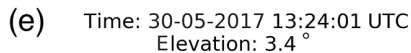

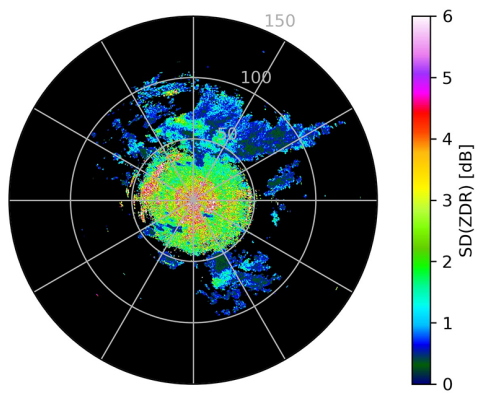

(b) Time: 30-05-2017 13:24:01 UTC

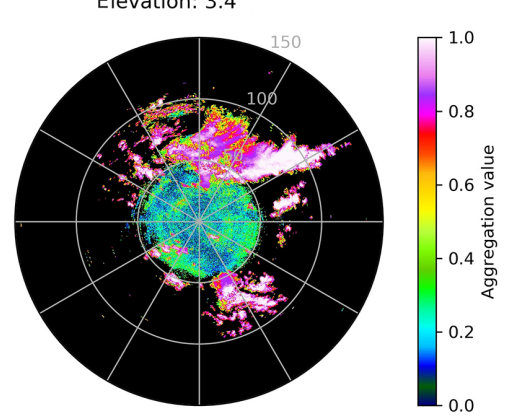

(d) Time: 30-05-2017 13:24:01 UTC

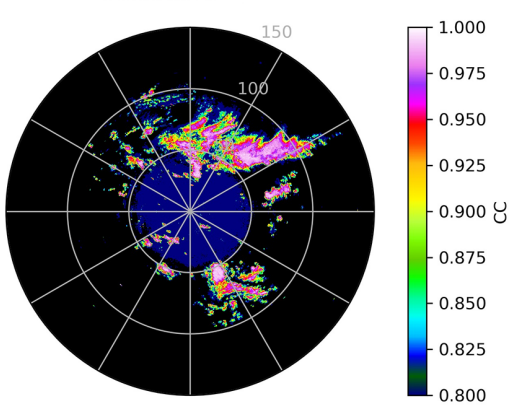

(f) Time: 30-05-2017 13:24:01 UTC

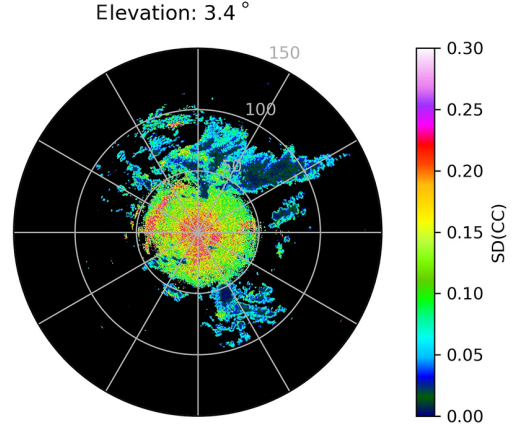

Figure 10. (a) $A_{\mathrm{MET}}$ (MetSignal_noise); (b) $A_{\mathrm{MET}}$ (MetSignal) after masking SNR less than $5 \mathrm{~dB}$; (c) ZDR after masking SNR less than $5 \mathrm{~dB}$; (d) CC after averaging along the radial using a $1.5 \mathrm{~km}$ window and masking SNR less than $5 \mathrm{~dB}$; (e) SD(ZDR) after masking SNR less than $5 \mathrm{~dB}$; and (f) $\mathrm{SD}(\mathrm{CC})$ after masking SNR less than $5 \mathrm{~dB}$. All from NUIST-CDP at 13:24 UTC on 30 May 2017 from an elevation of $3.4^{\circ}$.

(a) Time: 24-05-2017 23:53:48 UTC Elevation: $0.5^{\circ}$

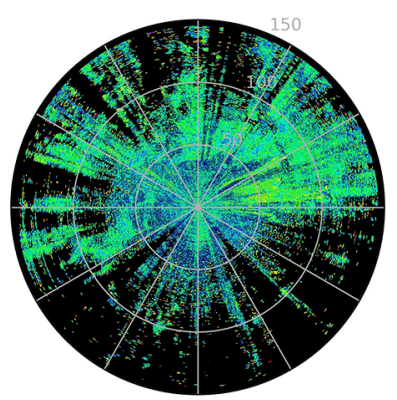

(b) Time: 24-05-2017 23:53:48 UTC Elevation: $0.5^{\circ}$
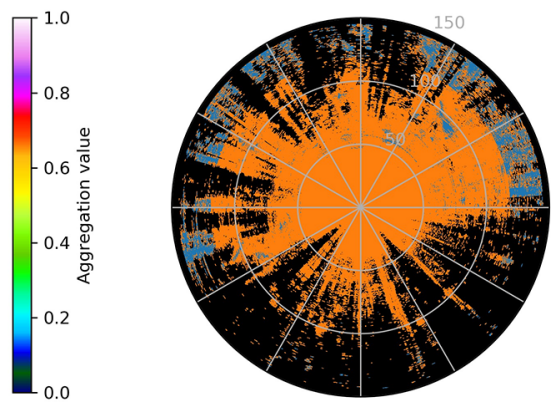

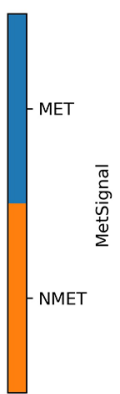

(c) Time: 24-05-2017 23:46:22 UTC

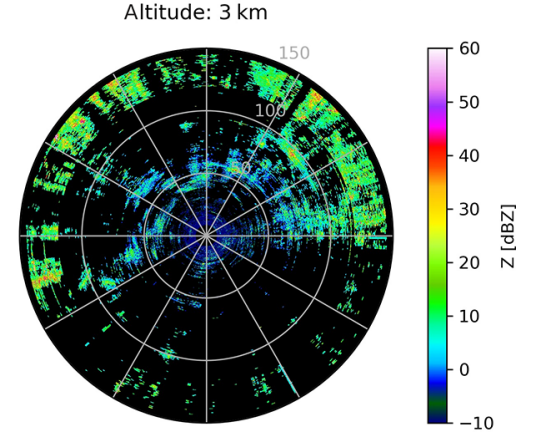

Figure 11. (a) $A_{\mathrm{MET}}$ (MetSignal); (b) MetSignal; and (c) the CAPPI of $Z$ at the altitude of $3 \mathrm{~km}$. (a, b) From NUIST-CDP at 23:53 UTC on 24 May 2017 from an elevation of $0.5^{\circ}$, while (c) is at 23:46 UTC on 24 May 2017 (the previous volume scan of a, b). 
in ML regions and NMET have similar characteristics in polarimetric features. Therefore, an additional fuzzy logic echo classifier, without the polarimetric features input, is implemented in the post-processing of the MetSignal_noise algorithm in the potential ML regions (initially defined as the regions over $2.5 \mathrm{~km}$ in height based on the statistical analysis using the training set), for range gates classified as NMET in the first fuzzy logic echo classifier. Considering that AP in the potential ML regions may not be classified effectively by solely using $Z$ and $V$, the $\operatorname{SD}(Z)$ (using the same estimation method as $\mathrm{SD}(\mathrm{ZDR})$ and $\mathrm{SD}(\mathrm{CC})$ ) is input into the additional fuzzy logic echo classifier, to improve the classification performance. Figure $2 \mathrm{f}$ shows the normalized frequency distribution and membership function of $\operatorname{SD}(Z)$, which are also derived from the training set, but these only use data in the potential ML regions. If these range gates (classified as NMET in the first fuzzy logic echo classifier in the potential ML regions) are classified as MET in the additional fuzzy logic echo classifier, then these are highly probable to be influenced by ML and should be reclassified as MET.

As shown in Fig. 12, NUIST-CDP observed a typical case of stratiform precipitation (10:49 UTC on 23 May 2017). Although the bright band characteristic of $Z$ is not very obvious (Fig. 12d), the location of the ML region at a range of about $100 \mathrm{~km}$ can be well revealed by ZDR and CC (Fig. 12e and f). As shown in Fig. 12b, $A_{\mathrm{MET}}$ before post-processing (the result of the first fuzzy logic echo classifier) has an obvious low value in the ML region, due to the similar characteristics of polarimetric features between MET in ML regions and NMET. $A_{\text {MET }}$ after post-processing (the range gates classified as NMET in the first fuzzy logic echo classifier will be substituted for $A_{\mathrm{MET}}$ of the additional fuzzy logic echo classifier) is shown in Fig. 12a; the abnormal decrease of $A_{\text {MET }}$ before the post-processing in the ML region is effectively suppressed. The final classification result (Fig. 12c), based on $A_{\text {MET }}$ after post-processing, shows good performance in the ML region.

In addition to the ML region, some other special MET cases with abnormal polarimetric features will also cause the misclassification of the algorithm (e.g., the threshold of the ZDR filter setting in the post-processing could be reached if wet hailstones are inside the radar sample volume). Therefore, the additional fuzzy logic echo classifier can also mitigate these problems to some extent by eliminating polarimetric features in the input.

\section{Evaluation}

To objectively evaluate the MetSignal_noise algorithm performance and its improvement compared with the MetSignal algorithm, a test set independent of the training set is selected and listed in Table 4 (the same extraction method as the training set). Two methods were used to compute skill: the fraction of correct classification (FCC) for each echo type and
Table 4. List of events used for evaluating algorithm performance (UTC).

\begin{tabular}{ll}
\hline Date & Description \\
\hline 04:00-05:00 21 May 2017 & CA \\
09:00-10:00 22 May 2017 & Convective precipitation \\
05:00-06:00 23 May 2017 & Stratiform precipitation \\
22:00-23:00 23 May 2017 & GC \\
22:00-23:00 24 May 2017 & AP \\
22:00-23:00 26 May 2017 & AP \\
12:00-13:00 28 May 2017 & CA \\
05:00-06:00 30 May 2017 & Stratiform precipitation \\
10:00-11:00 30 May 2017 & Convective precipitation \\
22:00-23:00 31 May 2017 & Stratiform precipitation \\
\hline
\end{tabular}

the overall Heidke skill score (HSS; Doswell III et al., 1990), which is computed as

$\mathrm{HSS}=\frac{2(a d-b c)}{(a+c)(c+d)+(a+b)(b+d)}$,

where $a$ represents the number of hits, $b$ represents the false alarms, $c$ represents the misses, and $d$ represents the correct nulls.

The skill results of the MetSignal and MetSignal_noise algorithms based on the test set are shown in Table 5. To facilitate the analysis of the dependence between the classification performance and SNR, the computation of the classification skill is stratified by three SNR intervals (larger than 15, 5-15, and larger than $5 \mathrm{~dB}$ ). By comparing the classification performance of the two algorithms in MET, it is found that the classification skill of the MetSignal_noise algorithm is significantly higher than that of the MetSignal algorithm, especially in the SNR interval greater than $5 \mathrm{~dB}$ and less than $15 \mathrm{~dB}$. This can verify that the MetSignal_noise algorithm can improve the classification performance of the MetSignal algorithm at a low SNR by stratifying polarimetric features based on SNR intervals and masking low SNR polarimetric variables in texture calculation. The better performance of the MetSignal_noise algorithm in the SNR interval greater than $15 \mathrm{~dB}$ is mainly owed to the fact that membership functions of polarimetric features are more targeted after SNR stratification. Compared with the difference of classification skill in MET between the two algorithms, the difference in NMET is smaller. The classification performance of the MetSignal algorithm in NMET is slightly better than that of the MetSignal_noise algorithm in the SNR interval greater than $5 \mathrm{~dB}$ and less than $15 \mathrm{~dB}$, which should be attributed to misclassification of CA into MET in the potential ML regions after postprocessing because non-polarimetric features (i.e., $Z, V$, and $\mathrm{SD}(Z)$ ) cannot effectively distinguish CA from MET (Lakshmanan et al., 2007; Tang et al., 2014). The main reason for the lower classification skill of the MetSignal algorithm in the SNR interval greater than $15 \mathrm{~dB}$ is that the $Z$ of AP is 
(a)

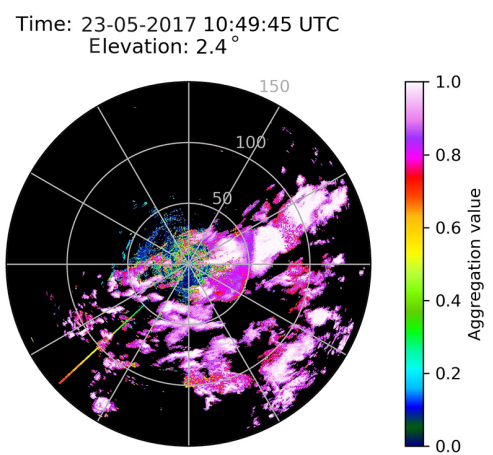

(c)

$$
\text { Time: 23-05-2017 10:49:45 UTC }
$$

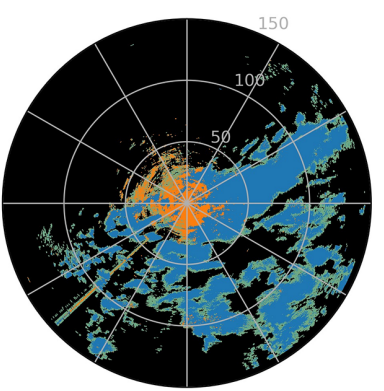

(e)
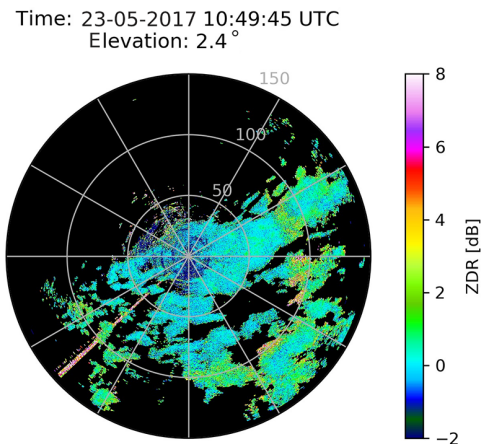

(b) Time: $23-05-2017$ 10:49:45 UTC

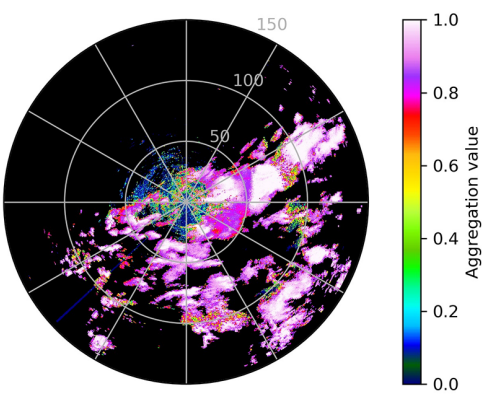

(d) Time: 23-05-2017 10:49:45 UTC

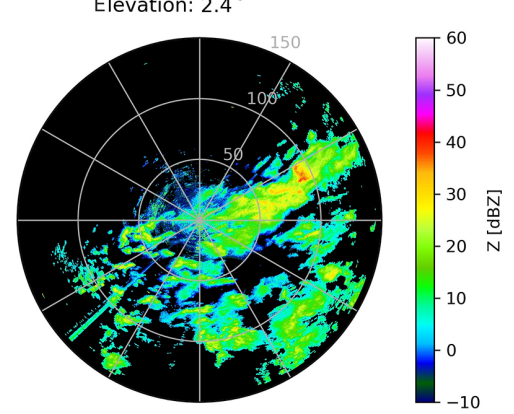

(f) Time: 23-05-2017 10:49:45 UTC

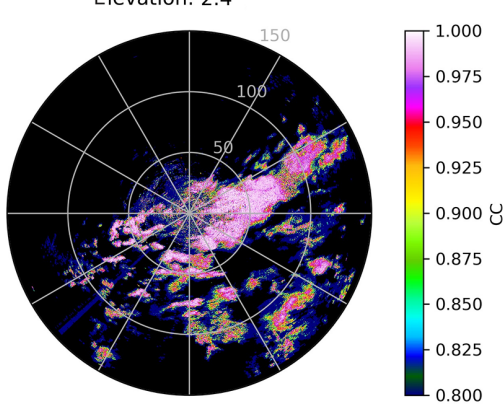

Figure 12. (a) $A_{\mathrm{MET}}$ (MetSignal_noise) after post-processing; (b) $A_{\mathrm{MET}}$ (MetSignal_noise) before post-processing; (c) MetSignal_noise; (d) Z; (e) ZDR; and (f) CC. All from NUIST-CDP at 10:49 UTC on 23 May 2017 from an elevation of $2.4^{\circ}$.

Table 5. The classification performance of MetSignal and MetSignal_noise algorithms, and the sensitivity analysis of different improvement steps in MetSignal_noise algorithms. All skill scores were computed based on data in the test set.

\begin{tabular}{|c|c|c|c|c|c|c|c|c|c|c|}
\hline & \multicolumn{3}{|c|}{ MetSignal } & \multicolumn{3}{|c|}{ MetSignal_noise } & \multicolumn{4}{|c|}{ Sensitivity analysis } \\
\hline & $\begin{array}{r}5<\text { SNR }>15 \\
(\mathrm{~dB})\end{array}$ & $\begin{array}{r}\text { SNR }>15 \\
(\mathrm{~dB})\end{array}$ & $\begin{array}{r}\text { SNR }>5 \\
(\mathrm{~dB})\end{array}$ & $\begin{array}{r}5<\mathrm{SNR}<15 \\
(\mathrm{~dB})\end{array}$ & $\begin{array}{r}\text { SNR }>15 \\
(\mathrm{~dB})\end{array}$ & $\begin{array}{r}\text { SNR > } 5 \\
(\mathrm{~dB})\end{array}$ & SA1 & SA2 & SA3 & SA4 \\
\hline $\mathrm{FCC}_{\mathrm{MET}}$ & $79.8 \%$ & $98.7 \%$ & $86.8 \%$ & $99.2 \%$ & $99.7 \%$ & $99.4 \%$ & $99.1 \%$ & $99.1 \%$ & $95 \%$ & $97.1 \%$ \\
\hline FCC $_{\text {NMET }}$ & $95.8 \%$ & $96.4 \%$ & $96.2 \%$ & $94.9 \%$ & $98.4 \%$ & $97.1 \%$ & $96.6 \%$ & $96.8 \%$ & $96.6 \%$ & $96.4 \%$ \\
\hline HSS & 0.756 & 0.95 & 0.83 & 0.94 & 0.981 & 0.965 & 0.957 & 0.959 & 0.916 & 0.935 \\
\hline
\end{tabular}

sometimes more than $11 \mathrm{dBZ}$ at $3 \mathrm{~km}$, and it will be classified as MET in the post-processing of the MetSignal algorithm.

In addition to evaluating the performance of the MetSignal and MetSignal_noise algorithms, it is also necessary to perform a sensitivity analysis of the four improvement steps in the MetSignal_noise algorithm, i.e., ignore one of the improvement steps and analyze its impact on classification performance. All sensitivity analyses were performed in the range of SNR larger than $5 \mathrm{~dB}$, and included four parts: SA1 (without the limitation of the use scope of $V$ ); SA2 (without 
the decrease of CC in the region of GC and AP); SA3 (without the improvements in the weak-signal region); and SA4 (without the adjustments of post-processing for the ML region). The results of the sensitivity analysis are summarized in Table 5. Compared with SA3 and SA4, SA1 and SA2 have less influence on the skill scores of the MetSignal_noise algorithm. The main reason is that the first two improvement steps mainly focus on NMET, but the classification performance of the MetSignal algorithm on NMET is very satisfactory (the difference of $\mathrm{FCC}_{\mathrm{NMET}}$ between the MetSignal algorithm and the MetSignal_noise algorithm is only $0.9 \%$ in the range of an SNR value larger than $5 \mathrm{~dB}$ ). In addition, the weights of $V$ and CC in the fuzzy logic echo classifier are small. Although the MetSignal_noise algorithm focuses on the improvement in the weak-signal region, SA3 does not have a substantial decrease in the skill scores compared to the MetSignal algorithm. The main reason is that the additional fuzzy logic echo classifier in the post-processing can correctly reclassify the misclassified MET in potential ML regions, where weak-signal echoes appear frequently. The decrease of FCC $_{\text {MET }}$ in SA4 is mainly attributed to misclassification in ML regions. However, due to the limited data affected by ML, the magnitude of the decrease is not very notable. The reason for the decrease of $\mathrm{FCC}_{\mathrm{NMET}}$ in SA4 is the same as the MetSignal algorithm; that is, some of AP is misclassified as MET after post-processing (MetSignal algorithm). From the HSS of the sensitivity analysis, it can be seen that ignoring any improvement step will reduce the overall score. Therefore, all the improvements have a positive effect on the classification performance, even if some improvements do not play an important role.

\section{Conclusions}

An improved fuzzy logic method, MetSignal_noise, is proposed in this paper to distinguish between MET and NMET using polarimetric radar data from NUIST-CDP. The most significant improvement over the raw version (MetSignal) is its better classification performance in weak-signal regions by stratifying polarimetric features based on SNR intervals and masking low SNR polarimetric variables in texture calculation. In addition, the thresholds of $Z$ and $W$ are set to limit the scope of $V$ in order to improve its classification performance and prevent its contribution to the misclassification of $\mathrm{CA}$. An averaging method along the radial is also used to decrease the abnormally high value of $\mathrm{CC}$ from $\mathrm{GC}$ and AP. The post-processing rule used to prevent misclassification in ML regions in the MetSignal algorithm sometimes reclassifies $\mathrm{AP}$ at a far range into MET; therefore, it has been removed in the MetSignal_noise algorithm and is substituted by an additional fuzzy logic echo classifier without the polarimetric features input.

An independent test set is selected to evaluate the algorithm performance; the results show that the MetSig- nal_noise algorithm is overall better than the MetSignal algorithm, especially in low SNR regions. However, the MetSignal_noise algorithm is slightly worse than the MetSignal algorithm in SNR intervals greater than $5 \mathrm{~dB}$ and less than $15 \mathrm{~dB}$. This is because some CA cases are reclassified as MET after post-processing because non-polarimetric features cannot effectively distinguish CA from MET. Although increasing the height threshold of the potential ML region can improve this defect as CA does not usually appear at high altitudes (Wilson et al., 1994), this will cause some low ML regions to miss the post-processing. At present, a CA identification method based on radial continuity is under development, which is expected to greatly reduce the risk of CA misclassification. The altitude of ML depends on the season and geographical location (Zhang and Qi, 2010). Therefore, real-time ML identification algorithms (Giangrande et al., 2008; Zhang et al., 2008) or atmospheric environmental data (Tang et al., 2014) have been considered as additions to the MetSignal_noise algorithm in the following study to select a better height threshold. Moreover, the advanced clutter suppression algorithm based on signal processing (Hubbert et al., 2009a, b; Torres and Warde, 2014) should be considered for introduction into NUIST-CDP. This is because when the ML appears at a lower altitude (frequently occuring in winter precipitation events), it will be fully mixed with the ground clutter. Then even if the height of ML is accurately located, the ML region may still be misclassified as NMET. The sensitivity analysis of the MetSignal_noise algorithm shows that all the improvements have a positive effect on the classification performance, even if some improvements are not significant. In addition, we plan to identify and eliminate the second-trip echo in a future work to further improve data quality.

Data availability. The radar data used in this study are available from the corresponding author upon request (huangxy@nuist.edu.cn).

Author contributions. SZ designed the methodology and prepared the original draft of the paper. $\mathrm{XH}$ and JM wrote and reviewed the paper. ZC and XZ validated the paper's finding. HZ edited the paper.

Competing interests. The authors declare that they have no conflict of interest.

Acknowledgements. We acknowledge the investigators and operators of NUIST-CDP. We also want to thank Chian Zhang of the Beijing Metstar Radar Company in China for providing constructive comments for this paper. 
Financial support. This research has been supported by the National Key Technologies R\&D Program of China (grant nos. 2017YFC1502102 and 2017YFC1502103) and the National Natural Science Foundation of China (grant no. 41430427).

Review statement. This paper was edited by Gianfranco Vulpiani and reviewed by two anonymous referees.

\section{References}

Bringi, V. N. and Chandrasekar, V.: Polarimetric Doppler Weather Radar, Cambridge University Press, Cambridge, UK, 2001.

Cho, Y. H., Lee, G., Kim, K. E., and Zawadzki, I.: Identification and Removal of Ground Echoes and Anomalous Propagation Using the Characteristics of Radar Echoes, J. Atmos. Ocean. Tech., 23, 1206-1222, 2006.

Crum, T. D. and Alberty, R. L.: The WSR-88D and the WSR-88D Operational Support Facility, B. Am. Meteorol. Soc., 74, 16691688, 1993.

Doswell III, C. A., Davies-Jones, R., and Keller, D. L.: On summary measures of skill in rare event forecasting based on contingency tables, Weather Forecast., 5, 576-585, 1990.

Doviak, R. J. and Zrnić, D. S.: Doppler radar and weather observations, Academic Press, Mineola, NY, 2nd edn., Dover Publications, Mineola, N.Y., 2006.

Fang, M., Doviak, R. J., and Melnikov, V.: Spectrum Width Measured by WSR-88D: Error Sources and Statistics of Various Weather Phenomena, J. Atmos. Ocean. Tech., 21, 888-904, 2004.

Giangrande, S. E. and Ryzhkov, A. V.: Estimation of Rainfall Based on the Results of Polarimetric Echo Classification, J. Appl. Meteorol. Clim., 47, 2445-2462, 2008.

Giangrande, S. E., Krause, J., and Ryzhkov, A. V: Automatic detection of the melting layer with a polarimetric prototype of the WSR-88D radar, J. Appl. Meteorol. Clim., 47, 1354-1364, 2008.

Gourley, J. J., Tabary, P., and Chatelet, J. P. D.: Data Quality of the Meteo-France C-Band Polarimetric Radar, J. Atmos. Ocean. Tech., 23, 1340-1356, 2006.

Gourley, J. J., Tabary, P., and Parent du Chatelet, J.: A Fuzzy Logic Algorithm for the Separation of Precipitating from Nonprecipitating Echoes Using Polarimetric Radar Observations, J. Atmos. Ocean. Tech., 24, 1439-1451, 2007.

Hubbert, J. C., Dixon, M., Ellis, S. M., and Meymaris, G.: Weather radar ground clutter. Part I: Identification, modeling, and simulation, J. Atmos. Ocean. Tech., 26, 1165-1180, 2009a.

Hubbert, J. C., Dixon, M., and Ellis, S. M.: Weather radar ground clutter. Part II: Real-time identification and filtering, J. Atmos. Ocean. Tech., 26, 1181-1197, 2009b.

Islam, T., Rico-Ramirez, M. A., Han, D., and Srivastava, P. K.: Artificial intelligence techniques for clutter identification with polarimetric radar signatures, Atmos. Res., 109-110, 95-113, 2012.

Jung, Y., Zhang, G., and Xue, M.: Assimilation of simulated polarimetric radar data for a convective storm using the ensemble Kalman filter. Part I: Observation Operators for Reflectivity and Polarimetric Variables, Mon. Weather Rev., 136, 2228-2245, 2008 a.
Jung, Y., Zhang, G., and Xue, M.: Assimilation of simulated polarimetric radar data for a convective storm using the ensemble Kalman filter. Part II: Impact of polarimetric data on storm analysis, Mon. Weather Rev., 136, 2246-2260, 2008 b.

Krause, J. M.: A Simple Algorithm to Discriminate between Meteorological and Nonmeteorological Radar Echoes, J. Atmos. Ocean. Tech., 33, 1875-1885, 2016.

Kumjian, M. R.: Principles and Applications of Dual-Polarization Weather Radar. Part I: Description of the Polarimetric Radar Variables, Journal of Operational Meteorology, 1, 226-242, 2013a.

Kumjian, M. R.: Principles and Applications of Dual-Polarization Weather Radar. Part II: Warm-and Cold-Season Applications, Journal of Operational Meteorology, 1, 243-264, 2013b.

Kumjian, M. R.: Principles and Applications of Dual-Polarization Weather Radar. Part III: Artifacts, Journal of Operational Meteorology, 1, 265-274, 2013c.

Lakshmanan, V., Fritz, A., Smith, T., Hondl, K., and Stumpf, G.: An Automated Technique to Quality Control Radar Reflectivity Data, J. Appl. Meteorol. Clim., 46, 288-305, 2007.

Lakshmanan, V., Karstens, C., Krause, J., and Tang, L.: Quality Control of Weather Radar Data Using Polarimetric Variables, J. Atmos. Ocean. Tech., 31, 1234-1249, 2014.

Lakshmanan, V., Karstens, C., Krause, J., Elmore, K., Ryzhkov, A., and Berkseth, S.: Which Polarimetric Variables Are Important for Weather/No-Weather Discrimination?, J. Atmos. Ocean. Tech., 32, 1209-1223, 2015.

Lin, P. F., Chang, P. L., Jou, J. D., Wilson, J. W., and Roberts, R. D.: Objective Prediction of Warm Season Afternoon Thunderstorms in Northern Taiwan Using a Fuzzy Logic Approach, Weather Forecast., 27, 1178-1197, 2012.

Liu, H. and Chandrasekar, V.: Classification of hydrometeors based on polarimetric radar measurements: Development of fuzzy logic and neuro-fuzzy systems, and in situ verification, J. Atmos. Ocean. Tech., 17, 140-164, 2000.

Park, H., Ryzhkov, A. V., Zrnic, D. S., and Kim, K. E.: The Hydrometeor Classification Algorithm for the Polarimetric WSR-88D: Description and Application to an MCS, Weather Forecast., 24, 730-748, 2009.

Rico-Ramirez, M. A. and Cluckie, I. D.: Classification of Ground Clutter and Anomalous Propagation Using Dual-Polarization Weather Radar, IEEE T. Geosci. Remote, 46, 1892-1904, 2008.

Ryzhkov, A. V., Giangrande, S. E., Melnikov, V. M., and Schuur, T. J.: Calibration Issues of Dual-Polarization Radar Measurements, J. Atmos. Ocean. Tech., 22, 1138-1155, 2005.

Schuur, T., Ryzhkov, A., Heinselman, P., Zrnic, D., Burgess, D., and Scharfenberg, K.: Observations and classification of echoes with the polarimetric WSR-88D radar, Report of the National Severe Storms Laboratory, Norman, OK, 73069, 46 pp., 2003.

Stumpf, G. J., Witt, A., Mitchell, D. W., Spencer, P. L., and Burgess, D. W.: The National Severe Storms Laboratory Mesocyclone Detection Algorithm for the WSR-88D, Weather Forecast., 13, 304326, 1998.

Tang, L., Zhang, J., Langston, C., Krause, J., Howard, K., and Lakshmanan, V.: A Physically Based Precipitation-Nonprecipitation Radar Echo Classifier Using Polarimetric and Environmental Data in a Real-Time National System, Weather Forecast., 29, 1106-1119, 2014. 
Torres, S. M. and Warde, D. A.: Ground clutter mitigation for weather radars using the autocorrelation spectral density, J. Atmos. Ocean. Tech., 31, 2049-2066, 2014.

Wang, Y. T. and Chandrasekar, V.: Algorithm for estimation of the specific differential phase, J. Atmos. Ocean. Tech., 26, 25652578, 2009.

Wilson, J. W., Weckwerth, T. M., Vivekanandan, J., Wakimoto, R. M., and Russell, R. W.: Boundary Layer Clear-Air Radar Echoes: Origin of Echoes and Accuracy of Derived Winds, J. Atmos. Ocean. Tech., 11, 1184-1206, 1994.

Zhang, J. and Qi, Y. C.: A real-time algorithm for the correction of brightband effects in radar-derived QPE, J. Hydrometeorol., 11, 1157-1171, 2010.

Zhang, J., Langston, C., and Howard, K.: Brightband Identification Based on Vertical Profiles of Reflectivity from the WSR-88D, J. Atmos. Ocean. Tech., 25, 1859-1872, 2008.
Zhang, J., Howard, K., Langston, C., Vasiloff, S., Kaney, B., Arthur, A., Cooten, S. V., Kelleher, K., Kitzmiller, D., and Feng, D.: National Mosaic and Multi-Sensor QPE (NMQ) System: Description, Results, and Future Plans, B. Am. Meteorol. Soc., 92, 13211338, 2011.

Zrnić, D. S., Ryzhkov, A., Straka, J., Liu, Y., and Vivekanandan, J.: Testing a Procedure for Automatic Classification of Hydrometeor Types, J. Atmos. Ocean. Tech., 18, 892-913, 2001.

Zrnić, D. S., Melnikov, V. M., and Ryzhkov, A. V.: Correlation Coefficients between Horizontally and Vertically Polarized Returns from Ground Clutter, J. Atmos. Ocean. Tech., 23, 381-394, 2006. 